Kragujevac Journal of Mathematics

Volume 39(1) (2015), Pages 93-109.

\title{
GEOMETRIC AND TOPOLOGICAL OBSTRUCTIONS TO VARIOUS IMMERSIONS IN SUBMANIFOLD THEORY AND SOME RELATED OPEN PROBLEMS
}

\author{
BANG-YEN CHEN ${ }^{1}$
}

\begin{abstract}
In this article we survey known results on geometric and topological obstructions to various important classes of isometric immersions in submanifold theory. In addition we present several related open problems. The main purpose of this article is an invitation for further research on the topics that it deals with.
\end{abstract}

\section{INTRODUCTION}

One very important problem in mathematics is to discover obstructions for various structures on manifolds. Here we quote two important results in this respect. First, R. Bott discovered in [4] a topological obstruction to integrability by proving that a subbundle $E$ of the tangent bundle $T M$ of a smooth manifold $M$ is integrable only if the ring $\operatorname{Pont}(T M / E)$ generated by the real Pontrjagin classes of $T M / E$ vanishes in dimensions greater than $2 \cdot \operatorname{dim}(T M / E)$. Another important result in this respect is due to A. Newlander and L. Nirenberg [47] which states that an almost complex manifold $(M, J)$ is a complex manifold if and only if the Nijenhuis tensor $N_{J}$ of $J$ vanishes identically. The latter result shows that the Nijenhuis tensor of the almost complex manifold provides an obstruction to the integrability of the almost complex structure on the almost complex manifold.

One of the most fundamental problems in the theory of submanifolds is the immersibility of a Riemannian manifold in a Euclidean space (or, more generally, in a space form). This immersion problem had been around since B. Riemann (1826-1866) and was posed explicitly by L. Schläfli (1814-1895) in the 1870s. According to the 1956 celebrated theorem of John F. Nash [46], every Riemannian manifold can be

Key words and phrases. Obstruction, $\delta$-invariants, minimal immersion, Lagrangian immersion, slant immersion, $C R$-immersion, finite type immersion.

2010 Mathematics Subject Classification. Primary: 53C42; Secondary 53C40.

Received: May 17, 2015

Accepted: May 22, 2015. 
isometrically immersed in some Euclidean spaces with sufficiently high codimension. In view of Nash's theorem, in order to investigate immersion problems in differential geometry it is very natural to impose some suitable conditions on the immersions.

In this article we survey known results on geometric and topological obstructions to various important classes of isometric immersions in submanifold theory. In addition we present several related open problems. The main purpose of this article is an invitation for further research on the topics that it deals with.

\section{Obstructions to minimal immersions}

We follow the notions and definitions from $[5,9,22,24]$. Throughout this article, by a compact manifold we mean a compact manifold without boundary.

Minimal surface theory originates with J. L. Lagrange (1736-1813) who in 1762 considered the variational problem of finding the surface $z=z(x, y)$ of least area stretched across a given closed contour. He derived the so-called Euler-Lagrange equation. Lagrange communicated his method in his first letter to L. Euler dated August 12, 1755 when he was only nineteen (cf. [38]). Since then the theory of minimal immersions have been investigated by many mathematicians in the last 250 years (see, e.g. [18, pages 207-242] and [48]).

In this section we discuss the following.

Problem 2.1. What are the obstructions for a Riemannian manifold to admit a minimal immersion in a Euclidean space?

2.1. Topological obstruction. Let $M$ be a Riemannian submanifold of a Euclidean $m$-space $\mathbb{E}^{m}$. A well-known result of E. Beltrami [2] states that the Laplacian $\Delta$ of $M$ satisfies

$$
\Delta \phi=-n H, \quad n=\operatorname{dim} M,
$$

where $\phi: M \rightarrow \mathbb{E}^{m}$ denotes the immersion and $H$ is the mean curvature vector.

Since the only harmonic functions on compact Riemannian manifolds are constant functions, it follows immediately from formula (2.1) that each minimal submanifold in a Euclidean space is non-compact. Consequently, "compactness" is a topological obstruction to minimal immersions into Euclidean spaces. To authors's knowledge this is the only known topological obstruction to general minimal immersions in Euclidean spaces.

2.2. Riemannian obstructions. For a Riemannian $n$-manifold $M$ isometrically immersed in a Euclidean space, the equation of Gauss states that the Riemann curvature tensor $R$ of $M$ satisfies

$$
R(X, Y ; Z, W)=\langle h(X, W), h(Y, Z)\rangle-\langle h(X, Z), h(Y, W)\rangle,
$$

where $\langle$,$\rangle and h$ denote the inner product and the second fundamental form of $M$, respectively. 
Let Ric and $\tau$ denote the Ricci curvature and scalar curvature of $M$, respectively. It follows from Gauss' equation (2.2) that a necessary condition for $M$ to admit a minimal immersion in a Euclidean space is that

$$
\text { Ric } \leq 0, \quad \text { in particular, } \tau \leq 0)
$$

with equality holding if and only if the submanifold is totally geodesic.

For many many years this was the only known Riemannian obstruction for a general Riemannian manifold to admit a minimal immersion into a Euclidean space with arbitrary codimension (before the invention of $\delta$-invariants). That is why S. S. Chern asked in the 1960s to search for further obstructions for a Riemannian manifold to admit a minimal immersion into a Euclidean space besides Ricci curvature.

In order to answer S. S. Chern's open problem, we need to introduce new types of Riemannian invariants, different in nature from the "classical" invariants; Ricci and scalar curvatures. Moreover, we also need to establish universal optimal relationships between the main extrinsic invariants; mainly the squared mean curvature $H^{2}$, with the new type of intrinsic invariants. These are the author's original motivation in 1990 's to introduce his so-called $\delta$-invariants on Riemannian manifolds.

Let us now recall the notion of $\delta$-invariants introduced in the 1990s (see [22] for details). Suppose that $M$ is a Riemannian $n$-manifold. Let $K(\pi)$ denote the sectional curvature of a plane section $\pi \subset T_{p} M$ of the tangent space $T_{p} M$ at $p \in M$.

For an orthonormal basis $e_{1}, \ldots, e_{n}$ of $T_{p} M$, the scalar curvature $\tau$ of $M$ at $p$ is defined by

$$
\tau(p)=\sum_{i<j} K\left(e_{i} \wedge e_{j}\right)
$$

Let $L$ be a $r$-subspace of $T_{p} M$ with $r \geq 2$. If $\left\{e_{1}, \ldots, e_{r}\right\}$ is an orthonormal basis of $L$, we then define the scalar curvature $\tau(L)$ of $L$ by

$$
\tau(L)=\sum_{\alpha<\beta} K\left(e_{\alpha} \wedge e_{\beta}\right), \quad 1 \leq \alpha, \beta \leq r .
$$

For two given integers $n, k$ with $n \geq 3$ and $k \geq 1$, we denote by $\mathcal{S}(n, k)$ the finite set consisting of $k$-tuples $\left(n_{1}, \ldots, n_{k}\right)$ of integers satisfying

$$
2 \leq n_{1}, \ldots, n_{k}<n, \quad n_{1}+\cdots+n_{k} \leq n .
$$

When $k=0$, we put $\mathcal{S}(n, k)=\varnothing$.

For each $k$-tuple $\left(n_{1}, \ldots, n_{k}\right) \in \mathcal{S}(n, k)$, the author introduced in the 1990s the following Riemannian invariant $\delta\left(n_{1}, \ldots, n_{k}\right)$ by

$$
\delta\left(n_{1}, \ldots, n_{k}\right)(p)=\tau(p)-\inf \left\{\tau\left(L_{1}\right)+\cdots+\tau\left(L_{k}\right)\right\}, \quad p \in M,
$$

where $L_{1}, \ldots, L_{k}$ run over all $k$ mutually orthogonal subspaces of $T_{p} M$ satisfying $\operatorname{dim} L_{j}=n_{j}, j=1, \ldots, k$. 
In particular, we have

$$
\begin{aligned}
\delta(\varnothing) & =\tau, \\
\delta(2)(p) & =\tau(p)-\inf K_{p}, \\
\delta(n-1) & =\max \operatorname{Ric}(p),
\end{aligned}
$$

where inf $K_{p}$ is the infinimum of the sectional curvature of $M$ at $p$.

The universal optimal inequality established in $[15,17]$ is the following (see also $[13,20,23])$.

Theorem 2.2. For any isometric immersion of a Riemannian n-manifold $M$ into a Euclidean space with arbitrary codimension, we have

$$
\delta\left(n_{1}, \ldots, n_{k}\right) \leq \frac{n^{2}\left(n+k-1-\sum_{j=1}^{k} n_{j}\right)}{2\left(n+k-\sum_{j=1}^{k} n_{j}\right)} H^{2}
$$

for each $k$-tuple $\left(n_{1}, \ldots, n_{k}\right) \in \mathcal{S}(n, k)$, where $H^{2}$ is the squared mean curvature.

An important immediate consequence of Theorem 2.2 is the following.

Theorem 2.3. Let $M$ be a Riemannian $n$-manifold. If there exist a point $p$ and a $k$-tuple $\left(n_{1}, \ldots, n_{k}\right) \in \mathcal{S}(n, k)$ with $\delta\left(n_{1}, \ldots, n_{k}\right)(p)>0$, then $M$ does not admit any minimal immersion into any Euclidean space for any codimension.

From Theorem 2.3 we know that each $\delta\left(n_{1}, \ldots, n_{k}\right)$ gives rise to a Riemannian obstruction to minimal immersions in Euclidean spaces. Consequently, we have many answers to Chern's open problem on minimal immersions.

Remark 2.1. The condition given in (2.3) is a special case of (2.8) due to (2.7).

Remark 2.2. If $M$ is a minimal surface in $\mathbb{E}^{3}$ with the induced metric $g$, then the Gauss curvature $G$ of $M$ is $\leq 0$. Thus $\sqrt{-G} g$ defines a new metric on points where $G \neq 0$. G. Ricci (1853-1925) proved in 1894 [50] that a given metric $g$ on a plane domain $D$ arises locally as the metric tensor of a minimal surface in $\mathbb{E}^{3}$ if and only if the Gauss curvature $G$ of $(D, g)$ is everywhere nonpositive and the corresponding Gauss curvature $\bar{G}$ of $\sqrt{-G} g$ vanishes at each point where $G \neq 0$. Let $g$ be the metric tensor of a minimal surface $M$ in $\mathbb{E}^{m}$. If $g$ satisfies Ricci's condition, then $g$ corresponds locally to the metric tensor of a minimal surface $\hat{M}$ in $\mathbb{E}^{3}$. H. B. Lawson proved in [45] that, in this case either $M$ lies in $\mathbb{E}^{3}$ and belongs to a specific oneparameter family of surfaces associated to $\hat{M}$, or else $M$ lies in $\mathbb{E}^{6}$ and it belongs to a specific two-parameter family of surfaces obtained from $\hat{M}$, none of which lie in any $\mathbb{E}^{5}$. Lawson's result implies that the Ricci condition is an intrinsic condition which completely characterizes minimal surfaces lying in $\mathbb{E}^{3}$ among all minimal surfaces in $\mathbb{E}^{4}$ or $\mathbb{E}^{5}$.

The Ricci condition provides an obstruction to minimal immersions of surfaces in $\mathbb{E}^{3}$. However, the Ricci condition only applies to minimal immersions of surfaces. 
Next, we gives some additional Riemannian obstructions to minimal immersions.

Theorem 2.4. [21] If a Riemannian manifold admits a non-trivial Riemannian submersion with totally geodesic fibers, then it cannot be isometrically immersed into any Riemannian manifold of non-positive curvature as a minimal submanifold.

It follows from Theorem 2.4 that "existence of a non-trivial Riemannian submersion structure with totally geodesic fibers" provides an additional geometric obstruction to minimal immersions in Euclidean spaces.

The next two results show that Riemannian manifolds admitting certain warped product structures also rise to obstructions to minimal immersions as well.

Theorem 2.5. [19] If a Riemannian manifold admits a warped product structure $M_{1} \times{ }_{f} M_{2}$ with harmonic warping function $f$, then it does not admit a minimal immersion into any Euclidean space for any codimension.

Theorem 2.6. [19] If $M_{1}$ is a compact Riemannian manifold, then every wared product $M_{1} \times{ }_{f} M_{2}$ does not admit a minimal immersion into any Riemannian manifold of negative sectional curvature. Also it does not admit a minimal immersion into any Euclidean space.

In view of these facts, I would like to propose the following.

Problem 2.7. Find further topological and geometric obstructions to minimal immersions.

\section{Obstructions to LAgrangian immersions}

Let $\tilde{M}^{n}$ be a Kähler $n$-manifold endowed with the complex structure $J$, a Kähler metric $g$ and the Kähler 2-form $\omega$. We denote by $\tilde{M}^{n}(4 c)$ a Kähler $n$-manifold with constant holomorphic sectional curvature $4 c$. We simply called such a Kähler manifold a complex space form.

It is well-known that a complete simply-connected complex space form $\tilde{M}^{n}(4 c)$ is holomorphically isometric to the complex Euclidean $n$-plane $\mathbb{C}^{n}$, the complex projective $n$-space $C P^{n}(4 c)$, or a complex hyperbolic $n$-space $C H^{n}(4 c)$ according to $c=0, c>0$ or $c<0$, respectively.

A totally real submanifold $M$ of a Kähler manifold $\tilde{M}$ (or more generally, of a Hermitian manifold) is a submanifold such that the almost complex structure $J$ of the ambient manifold $\tilde{M}$ carries each tangent space of $M$ into the corresponding normal space of $M$, that is, $J\left(T_{p} M\right) \subset T_{p}^{\perp} M$ for any point $p \in M$ (cf. [30]). Equivalently, $M$ is a totally real submanifold if and only if, for any nonzero vector $X$ tangent to $M$ at any point $p \in M$, the angle between $J X$ and the tangent plane $T_{p} M$ is equal to $\frac{\pi}{2}$. A totally real submanifold $M$ of a Kähler manifold $\tilde{M}$ is known as a Lagrangian submanifold if $\operatorname{dim}_{\mathbf{R}} M=\operatorname{dim}_{\mathbf{C}} \tilde{M}$; in other word, a Lagrangian submanifold is a totally real submanifold with the smallest possible codimension. 
A result of $\mathrm{M}$. Gromov states that a compact $n$-manifold $M$ admits a Lagrangian immersion (not necessary isometric) into the complex Euclidean $n$-space $\mathbb{C}^{n}$ if and only if the complexification $T M \otimes \mathbf{C}$ of the tangent bundle $T M$ of $M$ is trivial [41]. This result of Gromov implies that there are no topological obstructions to Lagrangian immersions for compact 3-manifolds in $\mathbb{C}^{3}$, simply due to the fact that the tangent bundle of every 3-manifold is trivial.

In addition, another result of Gromov [43] states that every compact embedded Lagrangian submanifold of $\mathbb{C}^{n}$ is not simply-connected (see [51] for a complete proof of this fact). This result is not true when the compact Lagrangian submanifolds were immersed but not imbedded. The simplest example is the Whitney immersion of an $n$-sphere $S^{n}$ in $\mathbb{C}^{n}$ defined by

$$
w\left(y_{0}, y_{1}, \ldots, y_{n}\right)=\frac{1+\mathrm{i} y_{0}}{1+y_{0}^{2}}\left(y_{1}, \ldots, y_{n}\right),
$$

with $y_{0}^{2}+y_{1}^{2}+\cdots+y_{n}^{2}=1$. The Whitney immersion $w: S^{n} \rightarrow \mathbb{C}^{n}$ is a Lagrangian immersion which has a unique self-intersection point at $w(-1,0, \ldots, 0)=w(1,0, \ldots, 0)$.

If one imposes isometrical condition on Lagrangian immersions, it leads to the next problem.

Problem 3.1. What are Riemannian obstructions to Lagrangian isometric immersions of a Riemannian $n$-manifold into $\mathbb{C}^{n}$ ?

In order to provide some answers to this problem, we recall the following result from [16].

Theorem 3.2. If a compact $n$-manifold $M, n \geq 2$, has finite fundamental group or null first Betti number, then every Lagrangian immersion of $M$ into any EinsteinKähler manifold has minimal points, i.e., the mean curvature vector of $M$ vanishes at some points on $M$.

An important consequence of Theorem 3.2 is the following (see [22, page 308]).

Corollary 3.1. There do not exist Lagrangian isometric immersions from a compact Riemannian manifold with positive Ricci curvature into any flat Kähler manifold or into any complex hyperbolic space.

Obviously, Corollary 3.1 provides a partial answer to Problem 3.1. Another solution to Problem 3.1 is the following obstruction obtained in [15, 17] (see also [22, page 308]).

Theorem 3.3. Let $M$ be a compact Riemannian $n$-manifold with null first Betti number or with finite fundamental group $\pi_{1}(M)$. If there exists a $k$-tuple $\left(n_{1}, \ldots, n_{k}\right) \in$ $\mathcal{S}(n, k)$ such that $\delta\left(n_{1}, \ldots, n_{k}\right)>0$, then $M$ does not admit any Lagrangian isometric immersion into $\mathbb{C}^{n}$. 


\section{Obstructions to SLANT imMERsions}

Let $M$ be a Riemannian $n$-manifold isometrically immersed in a Kähler manifold $(\tilde{M}, J, g)$. For a vector $X \in T M$, let $P X$ and $F X$ denote the tangential and the normal components of $J X$, respectively. For a nonzero vector $X \in T_{p} M$ at $p \in M$, the angle $\theta(X)$ between $J X$ and the tangent space $T_{p} M$ in $T_{p} \tilde{M}$ is called the Wirtinger angle of $X$.

A submanifold $M$ of $\tilde{M}$ is called a slant submanifold if the Wirtinger angle $\theta(X)$ is constant; so it is independent of the choice of $p \in M$ and of $X \in T_{p} M$. The Wirtinger angle of a slant submanifold is called the slant angle (cf. [11, 12]). Complex submanifolds and totally real submanifolds are nothing but slant submanifolds with slant angle $\theta=0$ and $\theta=\pi / 2$, respectively.

A slant submanifold is called proper slant if it is neither a complex submanifold nor a totally real submanifold. Clearly, every slant submanifold is even-dimensional unless it is totally real.

Analogous to Lagrangian immersions, it is natural to ask the following.

Problem 4.1. What are the obstructions to slant immersions of a Riemannian manifold into a complex Euclidean $m$-space $\mathbb{C}^{m}$ ?

We denote the $j$-th de Rham cohomology group of a manifold $M$ by $H^{j}(M ; \mathbf{R})$.

The next two theorems provide two very simple topological obstructions to slant immersions.

Theorem 4.2. [34] If $M$ is a compact $2 k$-manifold such that $H^{2 i}(M ; \mathbf{R})=\{0\}$ for some integer $i \in\{1, \ldots, k\}$, then $M$ cannot immersed into any Kähler manifold as a proper slant submanifold.

Theorem 4.3. [34] Every proper slant submanifold in a complex Euclidean space $\mathbb{C}^{m}$ is non-compact.

Remark 4.1. Although there do not exist compact proper slant submanifolds in some complex Euclidean spaces, there exist compact proper slant submanifolds immersed in some complex flat tori.

The next result from [15, 17] provides a Riemannian obstruction to slant immersions into flat Kähler manifolds.

Theorem 4.4. Let $M$ be a compact Riemannian n-manifold with finite fundamental group $\pi_{1}(M)$. If there is a $k$-tuple $\left(n_{1}, \ldots, n_{k}\right) \in \mathcal{S}(n, k)$ such that $\delta\left(n_{1}, \ldots, n_{k}\right)>0$ on $M$, then $M$ admits no slant immersion into any flat Kähler $n$-manifold.

Now, we propose the following.

Problem 4.5. Find further topological and geometric obstructions to Lagrangian and slant immersions, besides those given in this section. 


\section{Obstructions to $C R$-IMmersions}

A $C R$ manifold is a manifold together with a geometric structure modeled on that of a real hypersurface in a complex vector space. More precisely, let $N$ be a differentiable manifold and let $T^{\mathbf{C}} N$ denote the complexified tangent bundle of $N$. A $C R$ structure on $N$ is a complex subbundle $\mathcal{H}$ of $T^{\mathbf{C}} N$ such that $\mathcal{H} \cap \overline{\mathcal{H}}=\{0\}$ (i.e., $\mathcal{H}$ is almost Lagrangian) and $\mathcal{H}$ is integrable. A manifold endowed with a $C R$ structure is called a $C R$ manifold [40].

It is well-known that if $(N, \mathcal{H})$ is a $C R$ manifold, then there exists a unique (real) subbundle $\mathcal{D}$ of $T N$ and a field of endomorphism $\mathcal{J}: \mathcal{D} \rightarrow \mathcal{D}$ such that $\mathcal{J}^{2}=-I$. $\mathcal{D}$ is just $\operatorname{Re}(\mathcal{H} \oplus \overline{\mathcal{H}})$ and $\mathcal{H}_{p}=\left\{X-\mathrm{i} \mathcal{J} X: X \in \mathcal{D}_{p}\right\}$.

A Riemannian submanifold $M$ of a Hermitian manifold is called a $C R$-submanifold if there exist a holomorphic distribution $\mathcal{D}$ (i.e., $J\left(\mathcal{D}_{p}\right)=\mathcal{D}_{p}$ ) and a totally real distribution $\mathcal{D}^{\perp}$ (i.e., $J\left(\mathcal{D}_{p}^{\perp}\right) \subset T_{p}^{\perp} M$ ) on $M$ such that $T M=\mathcal{D} \oplus \mathcal{D}^{\perp}$ [1].

A $C R$-submanifold is called proper if it is neither a complex submanifold nor a totally real submanifold. Obviously, every real hypersurface $M$ of a Hermitian manifold with $\operatorname{dim}_{\mathbf{C}} M \geq 2$ is a $C R$-submanifold automatically.

Consider $C R$-immersions of a Riemannian manifold $M$, i.e., isometric immersions of $M$ in a Hermitian manifold immersed as a $C R$-submanifold. Assume that $M$ is a $C R$-submanifold of a Hermitian manifold $\tilde{M}$ with the holomorphic distribution $\mathcal{D}$. Let us consider the complex subbundle $\mathcal{L}$ of $T^{\mathbf{C}} M$ defined by $\mathcal{L}_{p}=\left\{X-\mathrm{i} \mathcal{J} X: X \in \mathcal{D}_{p}\right\}$.

It was proved by D. E. Blair and the author in [3] that $\mathcal{L}$ is a $C R$ structure on $M$. Thus every $C R$-submanifold of a Hermitian manifold is a $C R$ manifold. Consequently, for a manifold to admit a proper $C R$-immersion, it must admit a $C R$ structure.

There is another necessary condition for a manifold to admit a $C R$-immersion. In fact, it was proved in [6] that in order for a $C R$ manifold $M$ with $C R$ structure $\mathcal{H}$ to admit a $C R$-immersion in a Kähler manifold, the orthogonal complementary distribution $(\operatorname{Re}(\mathcal{H} \oplus \overline{\mathcal{H}}))^{\perp}$ of $\operatorname{Re}(\mathcal{H} \oplus \overline{\mathcal{H}})$ in $T M$ must be integrable.

Let $\mathcal{K}$ be a distribution of a Riemannian manifold $M$ with Levi-Civita connection $\nabla$. We denote the orthogonal complementary distribution of $\mathcal{K}$ in $T M$ by $\mathcal{K}^{\perp}$. Put

$$
\stackrel{\circ}{h}(X, Y)=\left(\nabla_{X} Y\right)^{\perp}
$$

for vector fields $X, Y$ in $\mathcal{K}$, where $\left(\nabla_{X} Y\right)^{\perp}$ denotes the $\mathcal{K}^{\perp}$-component of $\nabla_{X} Y$. Then $\grave{h}$ is a well-defined $\mathcal{K}^{\perp}$-valued tensor field. It follows from Frobenius' theorem that $\mathcal{K}$ is integrable if and only if $h$ is symmetric.

Let $e_{1}, \ldots, e_{k}$ be an orthonormal basis of $\mathcal{K}$. If we put

$$
\stackrel{\circ}{H}=\frac{1}{k} \operatorname{trace} \stackrel{\circ}{h}=\frac{1}{k} \sum_{j=1}^{k} \stackrel{\circ}{h}\left(e_{j}, e_{j}\right),
$$

then, up to sign, $\stackrel{\circ}{H}$ is a well-defined vector field, which is called the mean curvature vector of the distribution $\mathcal{K}$. If $\stackrel{\circ}{H}$ vanishes identically, then $\mathcal{K}$ is called a minimal distribution (cf. [22, page 200]). 
In addition to the two obstructions mentioned above, there exists a third obstruction to $C R$-immersions into Kähler manifolds. In fact, it was proved in [6] that in order for a $C R$ manifold $M$ to admits a $C R$-immersion in some Kähler manifold, the distribution $\operatorname{Re}(\mathcal{H} \oplus \overline{\mathcal{H}})$ must be a minimal distribution in $M$.

In view of these facts, we ask the problem.

Problem 5.1. Do there exist other obstructions to $C R$-immersions of a Riemannian manifold into a Kähler manifold besides the three obstructions given above?

\section{Obstructions to idEAL IMMERSiOnS}

Recall the following universal inequality from [15, 17, 20, 22].

Theorem 6.1. If $\phi: M \rightarrow N$ is an isometric immersion of a Riemannian n-manifold $M$ into a Riemannian $m$-manifold $N$, then we have

$$
\begin{aligned}
\delta\left(n_{1}, \ldots, n_{k}\right) \leq & \frac{n^{2}\left(n+k-1-\sum_{j=1}^{k} n_{j}\right)}{2\left(n+k-\sum_{j=1}^{k} n_{j}\right)} H^{2} \\
& +\frac{1}{2}\left\{n(n-1)-\sum_{j=1}^{k} n_{j}\left(n_{j}-1\right)\right\} \max K_{N}
\end{aligned}
$$

for each $k$-tuple $\left(n_{1}, \ldots, n_{k}\right) \in \mathcal{S}(n, k)$, where $\max K_{N}(p)$ denotes the maximum of the sectional curvature of the ambient space $N$ restricted to 2-plane sections of $T_{p} M$.

When the ambient space $N$ is the Euclidean $m$-space $\mathbb{E}^{m}$, Theorem 6.1 reduces to the following.

Theorem 6.2. If $\phi: M \rightarrow \mathbb{E}^{m}$ is an isometric immersion from a Riemannian $n$-manifold $M$ into $\mathbb{E}^{m}$, then we have

$$
\delta\left(n_{1}, \ldots, n_{k}\right) \leq \frac{n^{2}\left(n+k-1-\sum_{j=1}^{k} n_{j}\right)}{2\left(n+k-\sum_{j=1}^{k} n_{j}\right)} H^{2} .
$$

An isometric immersion $\phi: M \rightarrow \mathbb{E}^{m}$ is called $\delta\left(n_{1}, \ldots, n_{k}\right)$-ideal if it satisfies the equality case of (6.2) identically. Roughly speaking, ideal immersions are those immersions which receive the least possible tension from the ambient space (see [22, pages 268-269] for details).

Obviously, every negatively curved Riemannian manifold does not admit any ideal immersions in any Euclidean space.

Let us define a Riemannian invariant $\tilde{\Delta}_{0}$ on a Riemannian $n$-manifold $M$ by

$$
\tilde{\Delta}_{0}=\max \left\{\Delta\left(n_{1}, \ldots, n_{k}\right):\left(n_{1}, \ldots, n_{k}\right) \in \mathcal{S}(n, k) \text { and } k \geq 0\right\}
$$

where

$$
\Delta\left(n_{1}, \ldots, n_{k}\right)=\frac{2\left(n+k-\sum_{j=1}^{k} n_{j}\right)}{n^{2}\left(n+k-1-\sum_{j=1}^{k} n_{j}\right)} \delta\left(n_{1}, \ldots, n_{k}\right) .
$$


We recall the following result from [15, 17] (see also [22, Theorem 14.7]).

Theorem 6.3. A compact homogeneous Riemannian n-manifold $M$ with irreducible isotropy action admits an ideal immersion into a Euclidean space if and only if the first nonzero eigenvalue $\lambda_{1}$ of the Laplacian of $M$ satisfies $\lambda_{1}=n \hat{\Delta}_{0}$.

Not every compact homogeneous Riemannian manifold can be isometrically immersed in some Euclidean space as an ideal submanifold for some $k$-tuple $\left(n_{1}, \ldots, n_{k}\right)$. For example, although the unit sphere $S^{n}(1)$ does admit an ideal immersion in $\mathbb{E}^{n+1}$ as a hypersurface, Theorem 6.3 implies that the real, complex, quaternion projective spaces $R P^{n}, C P^{n}, Q P^{n}$ and the Cayley plane $\mathcal{O} P^{2}$ equipped with standard Riemannian metrics do not admit any ideal immersions into any Euclidean space.

In view of these facts, the following problem is natural and interesting.

Problem 6.4. Find topological and geometric obstructions to ideal immersions.

Theorem 6.3 provides a solution to Problem 6.4 for the class of compact irreducible homogeneous spaces. A more general solution for compact Riemannian manifolds is the following (see [17, Theorem 14.4(1)]).

Theorem 6.5. If the first non-zero eigenvalue $\lambda_{1}$ of the Laplacian $\Delta$ on a compact Riemannian $n$-manifold $M$ satisfies

$$
\lambda_{1}>\frac{n}{\operatorname{volume}(M)} \int_{M} \Delta\left(n_{1}, \ldots, n_{k}\right) d V
$$

for some $k$-tuple $\left(n_{1}, \ldots, n_{k}\right)$, then $M$ does not admit a $\delta\left(n_{1}, \ldots, n_{k}\right)$-ideal immersion into any Euclidean space.

A Riemannian manifold $M$ is called semi-symmetric if $R \cdot R=0$ holds identically, where $R \cdot R$ is defined by

$$
\begin{aligned}
(R(X, Y) \cdot R)(U, V) W:= & R(X, Y)(R(U, V) W)-R(R(X, Y) U, V) W \\
& -R(U, R(X, Y) V) W-R(U, V)(R(X, Y) W) \\
= & 0 .
\end{aligned}
$$

Given a symmetric $(0,2)$-tensor $B$ and any pair of vector field $X, Y$ on a Riemannian manifold $M$, one may consider the associated endomorphism:

$$
\left(X \wedge_{B} Y\right) Z:=B(Y, Z) X-B(X, Z) Y .
$$

The action of the natural metrical endomorphism $X \wedge_{g} Y$ as a derivation on the curvature tensor $R$ results the $(0,6)$-tensor $Q(g, R)=-\wedge_{g} \cdot R$, which is known as the Tachibana tensor.

A Riemannian manifold $M$ of dimension $\geq 3$ is called pseudo-symmetric (in the sense of Deszcz) if $R \cdot R=L \cdot Q(g, R)$ for some function $L$ on $M$. A Riemannian manifold is called Weyl semi-symmetric if $R \cdot C=0$, where $C$ denotes the Weyl conformal curvature tensor. Similarly, a Riemannian manifold is said to have pseudo-symmetric Weyl tensor if $C \cdot C=L_{C} Q(g, C)$ for a function $L_{C}$ on $M$. 
The following result was obtained in [37] by R. Deszcz, M. Petrović-Torgašev, L. Verstraelen and G. Zafindratafa.

Theorem 6.6. $A \delta(2)$-ideal submanifold $M$ of dimension $\geq 4$ in a Euclidean $m$-space $\mathbb{E}^{m}$ has pseudo-symmetric Weyl conformal curvature tensor $C$ and

$$
L_{C}=\frac{3-n}{(n-1)(n-2)} K_{\mathrm{inf}} .
$$

This theorem shows that a necessary condition for a Riemannian $n$-manifold $M$ with $n \geq 4$ to admit a $\delta(2)$-ideal immersion in some Euclidean space is that the manifold $M$ must have pseudo-symmetric Weyl conformal curvature tensor. Consequently, the Weyl curvature tensor $C$ gives rise to a geometric obstruction to $\delta(2)$-ideal immersions in Euclidean spaces.

\section{OBstRUCTIONS TO FINITE TYPE IMMERSIONS AND FINITE TYPE MAPS}

Let $\phi: M \rightarrow \mathbb{E}^{m}$ be a differentiable map from a Riemannian manifold $M$ into the Euclidean $m$-space $\mathbb{E}^{m}$. As before we denote the Laplacian of $M$ by $\Delta$. The map $\phi$ is said to be of finite type if $\phi$ is a finite sum of $\mathbb{E}^{m}$-valued eigen-maps of the Laplacian, i.e., if $\phi$ can be expressed as

$$
\phi=c+\phi_{1}+\phi_{2}+\cdots+\phi_{k}
$$

where $c$ is a constant vector in $\mathbb{E}^{m}$ and $\phi_{1}, \phi_{2}, \ldots, \phi_{k}$ are $k$ non-constant $\mathbb{E}^{m}$-valued maps satisfying

$$
\Delta x_{i}=\lambda_{i} x_{i}, i=1, \ldots, k .
$$

The decomposition (7.1) is called the spectral decomposition or the spectral resolution of $\phi$. In particular, if all of the eigenvalues $\lambda_{1}, \ldots, \lambda_{k}$ associated with the spectral decomposition are mutually different, then $\phi$ is said to be of $k$-type. If one of $\lambda_{1}, \ldots, \lambda_{k}$ in (7.2) is zero, then $\phi$ is said to be of null k-type (cf. $[9,31,14,24]$ for more details). Clearly, if a Riemannian manifold admits a null $k$-type map in a Euclidean space, then $M$ is non-compact.

A submanifold or a map is said to be of infinite type if it is not of finite type. In terms of finite type theory, a result of T. Takahashi [52] states that a submanifold of a Euclidean $m$-space $\mathbb{E}^{m}$ is of 1-type if and only if it is either a minimal submanifold of $\mathbb{E}^{m}$ or a minimal submanifold of a hypersphere of $\mathbb{E}^{m}$.

The class of finite type submanifolds is very large, which contains many important families of submanifolds; including all minimal submanifolds of Euclidean space; all minimal submanifolds of hyperspheres; all parallel submanifolds as well as all equivariantly immersed compact homogeneous submanifolds. In contrast, very few examples of finite type hypersurfaces are known.

Just like minimal submanifolds, submanifolds of finite type in a Euclidean space can be described by a spectral variation principle, namely as critical points of directional deformations [24, 27]. 
On one hand, the notion of finite type submanifolds provides a very natural way to combine the spectral geometry with the theory of submanifolds; as well as with maps; in particular with Gauss map. On the other hand, one can apply finite type theory to investigate spectral geometry of submanifolds. In fact, by studying finite type maps and finite type submanifolds, one often gets useful information on eigenvalues of a Riemannian manifold. By showing that a certain submanifold is of $k$-type, one can, in principle, determine $k$ eigenvalues of the Laplacian from the roots of its minimal polynomial.

The first results on finite type theory were collected in author's book [9] published in 1984. A report on the progress in this subject up to 1996 was presented in [14]. The most recent survey on finite type theory is the author's recent book [24].

7.1. Finite type immersions. The main purpose of this subsection is to discuss the following.

Problem 7.1. What are the obstructions to finite type immersions of a Riemannian manifold into a Euclidean space or a real space form (immersed standardly in a Euclidean space)?

In this subsection we concern mainly on this problem which are related with the following two conjectures on finite type hypersurfaces (see [14, page 321] and [24, page 180]).

Conjecture 7.2. The only compact hypersurfaces of finite type in a Euclidean space are hyperspheres.

Conjecture 7.3. The only complete non-compact hypersurfaces in a Euclidean space are standard hyperspheres, minimal hypersurfaces and hypersurfaces of null 2-type.

Now, we make some observations related to Problem 7.1 and Conjecture 7.2.

Observation 7.4. It is known that the only finite type immersions of a unit speed curve in the Euclidean 2-plane are open portions of circles and lines (cf. [24, Theorem 6.7]). Such curves are of 1-type. Consequently, there do not exist any obstruction to finite type immersions for 1-dimensional Riemannian manifolds.

In view of Observation 7.4 we shall only consider manifolds of dimension $n \geq 2$ throughout the remaining part of this section.

The next three observations illustrate that sectional curvature on a Riemannian manifold of dimension $n \geq 2$ rise gives to obstructions to finite type immersions in Euclidean spaces.

Observation 7.5. A classical result of D. Hilbert in [44] states that there do not exist complete surfaces in $\mathbb{E}^{3}$ with constant negative Gauss curvature. This result is false if the surfaces are non-complete. For example, the classical "pseudosphere" in $\mathbb{E}^{3}$ (generated by rotating a tractrix around a central axis) has constant Gauss curvature -1 . Hilbert's result implies that every complete surface of constant negative Gauss curvature admit no finite type immersions in $\mathbb{E}^{3}$. 
Observation 7.6. T. Otsuki [49] proved that every Riemannian $n$-manifold of constant negative sectional curvature cannot be isometrically immersed in $\mathbb{E}^{2 n-2}$. Therefore, for $n>2$, every Riemannian $n$-manifold with constant negative curvature admits no finite type immersions in $\mathbb{E}^{n+1}$.

For compact manifolds, we make the following.

Observation 7.7. Every compact Riemannian $n$-manifold $M$ with sectional curvature $K \leq 0$ and $n \geq 2$ admits no finite type immersions in $\mathbb{E}^{n+1}$.

Proof. Assume that $\psi: M \rightarrow \mathbb{E}^{n+1}$ is an isometric immersion of a compact Riemannian $n$-manifold $M$ into $\mathbb{E}^{n+1}$. Without loss of generality, we may assume that $M$ is orientable; otherwise we can replace $M$ by its two-fold covering.

Let $e$ be a unit normal vector of $M$ in $\mathbb{E}^{n+1}$. Consider the height function $h_{e}$ defined on $M$ by $h_{e}(p)=\langle e, \psi(p)\rangle, p \in M$. Then we have $X h_{e}=\langle e, X\rangle$ for each vector $X \in T M$. Thus we get

$$
X Y h_{e}=\left\langle e, \nabla_{X} Y+h(X, Y)\right\rangle
$$

for $X, Y$ tangent to $M$. Since $h_{e}$ is a continuous function on a compact manifold, $h_{e}$ has at least one absolute maximum, say at $q$. Because $e$ is normal to $M$ at $q$, we find from (7.3) that the shape operator $A$ of $M$ satisfies

$$
X Y h_{e}=\left\langle A_{e} X, Y\right\rangle
$$

at $q$. Hence the shape operator $A$ is positive definite at $p$. Consequently, the sectional curvature of $M$ cannot be $\leq 0$ at $p$.

Remark 7.1. Observation 7.7 is false when the codimension of $M$ in $\mathbb{E}^{m}$ is $\geq 2$. The simplest example is the standard imbedding of the flat torus $S^{1}(1) \times S^{1}(1) \subset \mathbb{E}^{4}$ which is of 1-type.

For finite type spherical immersions, we mention the following.

Theorem 7.8. [25] If $M$ is a compact surface with constant negative Gauss curvature. Then $M$ admits no finite type immersions into $S^{3}(1)$.

Theorem 7.8 shows that Gauss curvature gives rise to an obstruction for finite type immersions of a compact surface in $S^{3}(1) \subset \mathbb{E}^{4}$.

Contrast to Theorem 7.8, there do exist finite type immersions of compact surfaces with constant Gauss curvature $\geq 0$. For instance, a totally geodesic $S^{2}(1) \subset S^{3}(1)$ and the Clifford torus $T^{2}=S^{1}(1 / \sqrt{2}) \times S^{1}(1 / \sqrt{2}) \subset S^{3}(1)$ are 1-type immersions in $S^{3}(1) \subset \mathbb{E}^{4}$.

7.2. Obstructions to submanifold with low type Gauss map. Let $V$ be a linear $n$-subspace of $\mathbb{E}^{m}$. If $e_{n+1}, \ldots, e_{m}$ is an oriented orthonormal basis of $V$, then $e_{n+1} \wedge \cdots \wedge e_{m}$ is a decomposable $(m-n)$-vector of norm one and $e_{n+1} \wedge \cdots \wedge e_{m}$ gives an orientation of $V$. Conversely, each decomposable $(m-n)$-vector of norm one determines a unique oriented linear $n$-subspace of $\mathbb{E}^{m}$. 
If $G(m, m-n)$ denotes the Grassmann manifold of oriented linear $(m-n)$-subspaces in $\mathbb{E}^{m}$, we may then identify $G(m, m-n)$ with the set of decomposable $(m-n)$ vectors of norm one. Hence $G(m, m-n)$ can be regarded as an $n(m-n)$-dimensional submanifold of the unit hypersphere $S^{N_{m, n}-1}(1)$ centered at the origin in $=\wedge^{m-n} \mathbb{E}^{m}$ in a natural way. Therefore we have the following canonical inclusions:

$$
G(m, m-n) \subset S^{N_{m, n}-1}(1) \subset \mathbb{E}^{N_{m, n}}:=\wedge^{m-n} \mathbb{E}^{m}, \quad N_{m, n}=\left(\begin{array}{c}
m \\
n-m
\end{array}\right) .
$$

The classical Gauss map was introduced by Gauss in his fundamental paper [39] on surfaces. He used the classical Gauss map to define Gauss curvature. Since then the notion of Gauss map plays one of important roles in the theory of submanifolds.

For a submanifold $M$ of $\mathbb{E}^{m}$ with arbitrary codimension, the classical Gauss map $\hat{\nu}$ can be defined as the map

$$
\hat{\nu}: M \rightarrow G(m, m-n), \quad n=\operatorname{dim} M,
$$

which carries a point $p \in M$ to the linear $(m-n)$-subspace of $\mathbb{E}^{m}$ obtained from the normal space $T_{p}^{\perp} M$ via parallel displacement. If $\left\{e_{n+1}, \ldots, e_{m}\right\}$ is an oriented orthonormal frame of $T^{\perp} M$, then the Gauss map:

$$
\nu: M \rightarrow G(m, m-n) \subset S^{N_{m, n}-1}(1) \subset \mathbb{E}^{N_{m, n}}
$$

is defined by $\nu(p)=\left(e_{n+1} \wedge \cdots \wedge e_{m}\right)(p)$.

In this subsection we are concerning the following.

Problem 7.9. What are the obstructions to isometric immersions of a Riemannian manifold into a Euclidean space with finite type (or low type) Gauss map?

Analogous to Observation 7.4, there are no obstructions to curves in 1-type Gauss map (see [32, Proposition 3.1]). Hence in order to study Problem 7.9 it is natural to assume that the submanifold is of dimension $\geq 2$.

Ordinary spheres and square tori do admit immersions with 1-type Gauss map. Otherwise, very little were known on Problem 7.9. However, we have the following result which is an immediate consequence of [32, Theorem 4.4].

Theorem 7.10. Each compact surface with genus $\geq 2$ admits no immersions into any Euclidean space with 1-type Gauss map.

In addition to Theorem 7.10, we have another topological obstruction to immersions with low type Gauss map.

Theorem 7.11. [29] Let $M$ be a compact $n$-manifold with nonzero Euler number. Then $M$ does not admit a Lagrangian immersion into $\mathbb{C}^{n}$ whose Gauss map has type number $<n / 2$.

Remark 7.2. In views of these facts, it is interesting to obtain further obstructions for a submanifold in a Euclidean space with low type Gauss map. 


\section{REFERENCES}

[1] A. Bejancu, CR-submanifolds of a Kaehler manifold I, Proc. Amer. Math. Soc. 69 (1978), 134-142.

[2] E. Beltrami, Ricerche di analisi applicata alla geometria, Giornale di Math. II (1864), 150-162.

[3] D. E. Blair and B.-Y. Chen, On CR-submanifolds of Hermitian manifolds, Israel J. Math. 34 (1979), 353-363.

[4] R. Bott, On a topological obstruction to integrability, 1970 Global Analysis (Proc. Sympos. Pure Math., 16, Berkeley, Calif., 1968) pp. 127-131.

[5] B.-Y. Chen, Geometry of submanifolds, Marcel Dekker, New York, 1973.

[6] B.-Y. Chen, CR-submanifolds of a Kaehler manifold I, J. Differential Geom. 16 (1981), no. 2, 305-322.

[7] B.-Y. Chen, CR-submanifolds of a Kaehler manifold II, J. Differential Geom. 16 (1981), no. 3, 493-509.

[8] B.-Y. Chen, Cohomology of CR-submanifolds, Ann. Fac. Sc. Toulouse Math. Ser. V, 3 (1981), $167-172$.

[9] B. Y. Chen, Total Mean Curvature and Submanifolds of Finite Type, World Scientific, 1984.

[10] B. Y. Chen, Finite type submanifolds and generalizations, University of Rome, 1985.

[11] B.-Y. Chen, Slant immersions, Bull. Austral. Math. Soc. 41 (1990), no. 1, 135-147.

[12] B.-Y. Chen, Geometry of slant submanifolds, Katholieke Universiteit Leuven, 1990.

[13] B.-Y. Chen, Some pinching and classification theorems for minimal submanifolds, Arch. Math. 60 (1993), 568-578.

[14] B. Y. Chen, A report on submanifolds of finite type, Soochow J. Math. 22 (1996), 117-337.

[15] B.-Y. Chen, Strings of Riemannian invariants, inequalities, ideal immersions and their applications, The Third Pacific Rim Geometry Conference (Seoul, 1996), 7-60, Intern. Press, 1998.

[16] B.-Y. Chen, A vanishing theorem for Lagrangian immersions into Einstein-Kaehler manifolds and its applications, Soochow J. Math. 24 (1998), no. 2, 155-162.

[17] B.-Y. Chen, Some new obstruction to minimal and Lagrangian isometric immersions, Japan. J. Math. 26 (2000), 105-127.

[18] B.-Y. Chen, Riemannian submanifolds, Handbook of Differential Geometry, vol. I (2000), (eds. F. Dillen and L. Verstraelen), 187-418.

[19] B.-Y. Chen, On isometric minimal immersions from warped products into real space forms, Proc. Edinb. Math. Soc. 45 (2002), no. 3, 579-587.

[20] B.-Y. Chen, A general optimal inequality for arbitrary Riemannian submanifolds, J. Inequal. Pure Appl. Math. 6 (2005), no. 3, Paper No. 77, 10 p.

[21] B.-Y. Chen, Riemannian submersions, minimal immersions and cohomology class, Proc. Japan Acad. Ser. A Math. Sci. 81 (2005), 162-167.

[22] B.-Y. Chen, Pseudo-Riemannian geometry, $\delta$-invariants and Applications, World Scientific, Hackensack, NJ, 2011.

[23] B.-Y. Chen, A tour through $\delta$-invariants: From Nash embedding theorem to ideal immersions, best ways of living and beyond, Publ. Inst. Math. (Beograd) (N.S.), 94(108) (2013), 67-80.

[24] B.-Y. Chen, Total mean curvature and submanifolds of finite type, 2nd Edition, World Scientific, Hackensack, NJ, 2015.

[25] B.-Y. Chen and F. Dillen, Surfaces of finite type and constant curvature in the 3-sphere, C. R. Math. Rep. Acad. Sci. Canada, 12, 47-49.

[26] B.-Y. Chen and F. Dillen, Optimal general inequalities for Lagrangian submanifolds in complex space forms, J. Math. Anal. Appl. 379 (2011), 229-239.

[27] B. Y. Chen, F. Dillen, L. Verstraelen and L. Vrancken, A variational minimal principle characterizes submanifolds of finite type, C.R. Acad. Sc. Paris 317 (1993), 961-965. 
[28] B.-Y. Chen, F. Dillen, J. Van der Veken and L. Vrancken, Curvature inequalities for Lagrangian submanifolds: the final solution, Differential Geom. Appl. 31 (2013), no. 6, 808-819.

[29] B.-Y. Chen, J.-M. Morvan and T. Nore, Energy, tension and finite type maps, Kodai Math. J. 9 (1986), 406-418.

[30] B.-Y. Chen and K. Ogiue, On totally real submanifolds, Trans. Amer. Math. Soc. 193 (1974), $257-266$.

[31] B.-Y. Chen and M. Petrovic, On spectral decomposition of immersions of finite type, Bull. Austral. Math. Soc. 44 (1991), no. 1, 117-129.

[32] B.-Y. Chen and P. Piccinni, Submanifolds with finite type Gauss map, Bull. Austral. Math. Soc. 44 (1987), 161-186.

[33] B.-Y. Chen and Y. Tazawa, Slant surfaces of codimension two, Ann. Fac. Sci. Toulouse Math. 11 (1990), no. 3, 29-43.

[34] B.-Y. Chen and Y. Tazawa, Slant submanifolds in complex Euclidean spaces, Tokyo J. Math. 14 (1991), no. 1, 101-120.

[35] B.-Y. Chen and Y. Tazawa, Slant submanifolds of complex projective and complex hyperbolic spaces, Glasgow Math. J. 42 (2000), no. 3, 439-454.

[36] S. S. Chern, Minimal Submanifolds in a Riemannian Manifold, University of Kansas, 1968.

[37] R. Deszcz, M. Petrović-Torgašev, L. Verstraelen and G. Zafindratafa, On the intrinsic symmetries of Chen ideal submanifolds, Bull. Transilv. Univ. Brasov Ser. III, 1(50) (2008), 99-108.

[38] L. Euler, Euler à Lagrange, Berolini, die 6 Sept. 1755, Oeuvres de Lagrange 14 (1755), 144-146.

[39] C. F. Gauss, Disquisitiones generales circa superficies curvas, Comment. Soc. Sci. Gotting. Recent. Classis Math. 6 (1827).

[40] S. Greenfield, Cauchy-Riemann equations in several variables, Ann. Scoula Norm. Sup. Pisa 22 (1968), 275-314

[41] M. Gromov, A topological technique for the construction of solutions of differential equations and inequalities, Intern. Congr. Math. (Nice 1970) 2 (1971), 221-225.

[42] M. Gromov, Isometric immersions of Riemannian manifolds, Elie Cartan et les Mathématiques d'Aujourd'hui, Astérisque 1985, pp. 129-133.

[43] M. Gromov, Pseudoholomorphic curves in symplectic manifolds, Invent. Math. 82 (1985), 307347.

[44] D. Hilbert, Ueber Flächen von konstanter Gausscher Krümmung, Trans. Amer. Math. Soc. 2 (1901), 87-99.

[45] H. B. Lawson, Some intrinsic characterizations of minimal surfaces, J. D'Analyse Math. 24 (1971), 151-161.

[46] J. F. Nash, The imbedding problem for Riemannian manifolds, Ann. of Math. 63 (1956), 20-63.

[47] A. Newlander and L. Nirenberg, Complex analytic coordinates in almost complex manifolds, Annals of Math. 65 (1957), 391-404.

[48] J. C. C. Nitschem Lectures on minimal surfaces, Cambridge Univ. Press, 1989.

[49] T. Otsuki, Isometric imbedding of Riemann manifolds in a Riemann manifold, J. Math. Soc. Japan 6, (1954), 221-234.

[50] G. Ricci, Sulla teoria intrinseca delle superficie ed in ispecie di quelle di secondo, Atti R. Ist. Ven. di Lett. ed Arti. 6 (1894), 445-488.

[51] J. Sikorav, Non-existence de sous-variété lagrangienne exacte dans $\mathbf{C}^{n}$ (d'après Gromov), Aspects Dynamiques et Topologiques des Groupes Infinis de Transformation de la Mécanique Lyon, Travaux en Cours, Hermann, Paris 25 (1986), 95-110.

[52] T. Tahakashi, Minimal immersions of Riemannian manifolds, J. Math. Soc. Japan 18 (1966), $380-385$. 
${ }^{1}$ Department of Mathematics,

Michigan State University,

East Lansing, Michigan 48824-1027, U.S.A.

E-mail address: bychen@math.msu.edu 